\title{
8-Bit Sub Threshold Ripple Carry Adders in 32nm CMOS Technology for Wireless Sensor nodes.
}

\author{
R. Savari Rani Dr.C.Christober Asir Rajan . V.HARINI
}

\begin{abstract}
Subthreshold design has been proposed in the literature as an effective technique for designing signal processing circuits needed in wireless sensor nodes powered by sources with limited energy. The full adder cell forms the basic building block of majority of these signal processing circuits. In this paper, 8-Bit subthreshold Ripple Carry Adders (RCAs) for wireless sensor nodes optimized for ultra low power operation are proposed. Major contribution of this work is conversion of BSIM4, Predictive Technology Model (32nm) to EKV model ( charge based model). The 8-bit RCAs are simulated with HSPICE (Level =55) using the 32nm CMOS technology at supply voltages ranging from $0.25 \mathrm{~V}$ to $0.4 \mathrm{~V}$. Various metrics such as delay, average power and power delay product (PDP) are simulated and reported for effective twelve different topologies. The circuit designers can choose the full adder topology and the supply voltage that is suitable for their applications. Usage of EKV models results in 11\% reduction in power than that of using BSIM models for the adder cell "CB" with supply voltage of $0.2 \mathrm{~V}$.
\end{abstract}

The full text of the article is not available in the cache. Kindly refer the IJCA digital library at www.ijcaonline.org for the complete article. In case, you face problems while downloading the full-text, please send a mail to editor at editor@ijcaonline.org 